\title{
Perpetual convertible bonds in jump-diffusion models
}

\author{
Pavel V. Gapeev Christoph Kühn
}

\begin{abstract}
A convertible (callable) bond is a security that the holder can convert into a specified number of underlying shares. In addition, the issuer can recall the bond, paying some compensation, or force the holder to convert it immediately. We give an explicit solution to the corresponding optimal stopping game in the context of a reduced form model driven by a Brownian motion and a compound Poisson process with exponential jumps. It turns out that the occurrence of jumps leads to optimal stopping strategies whose structure differs from the results for continuous models.
\end{abstract}

Key words: Convertible bond, optimal stopping game, jump-diffusion model, integro-differential free-boundary problem, continuous and smooth fit, Itô-Tanaka-Meyer formula. Mathematics Subject Classification (2000): Primary 60G40, 34K10, 91B28. Secondary 60J60, 60J75.

\section{Introduction}

The market for convertible bonds has been growing rapidly during the last years. A convertible bond can be considered as a hybrid between a standard corporate bond and a stock. To issue a convertible bond is especially attractive for relatively small firms with high growth potential and risk. Such firms can often pay only small coupons but are willing to compensate this by participating the holder in the possible rise of their stocks.

Convertible bonds have been investigated rather extensively. Especially quite recently the corresponding optimal stopping problems have attracted much attention in the literature on mathematical finance. One has to distinguish between reduced form models where 
the stock price process of the issuing firm is directly given by some stochastic process and structural models where the starting point is the firm value which is the sum of the total equity value and the total debt value. Within a firm value model the pricing problem is treated in Sîrbu, Pikovsky and Shreve [31]. In contrast to earlier articles of Brennan and Schwartz [4] and Ingersoll [11]-[12], their paper includes the case where an earlier conversion of the bond can be optimal. This fact necessitates to address a nontrivial freeboundary problem. In the present article we work with reduced form models where such a contract can be expressed as a standard game contingent claim (see Davis and Lischka [6] for a complete introduction and a precise description of the contract). The arbitrage-free prices for a game contingent claim are given by the values of the corresponding zero-sum optimal stopping games considered under some martingale measures (see Kifer [17] for complete market models, and Kallsen and Kühn [16] for the incomplete case). In addition, by utility arguments one can justify a special choice for the martingale measure in incomplete models (see, e.g., Kallsen and Kühn [15]). Explicit solutions for the perpetual put option of game type are obtained in Kyprianou [21].

The special feature of this paper is that we do not restrict ourselves to the classical Black-Scholes model, but study a more general jump-diffusion model, where aiming at closed form solutions we consider the perpetual case and let the jumps be exponentially distributed. Besides the analytical tractability of this model, it has some other desirable properties. For example, it is able to reproduce the leptokurtic feature of the return distribution. In addition, taking a HARA-type utility function and the corresponding utility-based martingale measure, the jumps remain exponentially distributed under the measure transformation (see Kou [18] and Kou and Wang [19] for a detail description of the model). Therefore, our model can describe the stochastic dynamics under some economically justified martingale measure. From observed plain-vanilla option prices the parameters $\lambda$ and $\theta$ in the formula (2.1) below can be calibrated, which specifies the martingale measure.

It turns out that the occurrence of jumps in the model may change the structural behavior of the solution, and the issuer of a convertible bond is affected by the risk that the conversion value can jump over the recall price. Moreover, we can observe some phenomena 
which are quite unusual in optimal stopping problems. This is in contrast to standard American put and call options, where it was shown that the structure of optimal exercise times does not change under an extension of the driving process from Brownian motion to a more general Lévy process (see, e.g., Mordecki [23]-[24]). However, the phenomena observed in this paper should correspondingly also hold in the non-perpetual case as well as in more general jump-diffusion models.

The paper is organized as follows. In Section 2 we formulate the corresponding optimal stopping game and reduce it to an equivalent integro-differential double free-boundary problem. In Section 3 we derive an explicit solution to the free-boundary problem that also prepares the proof of the main result which is stated in Theorem 4.1. In Section 4 we verify that the solution of the free-boundary problem turns out to be a solution of the initial optimal stopping problem. In Section 5 we give some concluding remarks and comment the structure of the solution under different relationships on the parameters of the model.

\section{Formulation of the problem}

For a precise formulation of the problem let us consider a probability space $(\Omega, \mathcal{F}, P)$ with a standard Brownian motion $W=\left(W_{t}\right)_{t \geq 0}$ and a jump process $J=\left(J_{t}\right)_{t \geq 0}$ defined by $J_{t}=\sum_{i=1}^{N_{t}} \xi_{i}$, where $N=\left(N_{t}\right)_{t \geq 0}$ is a Poisson process with intensity $\lambda$ and $\left(\xi_{i}\right)_{i \in \mathbb{N}}$ is a sequence of independent random variables exponentially distributed with parameter 1 $\left(W, N\right.$ and $\left(\xi_{i}\right)_{i \in \mathbb{N}}$ are supposed to be independent). The stock price process $S=\left(S_{t}\right)_{t \geq 0}$ is given by

$$
S_{t}=s \exp \left(\left(r-\delta-\frac{\eta^{2}}{2}-\frac{\lambda \theta}{1-\theta}\right) t+\eta W_{t}+\theta J_{t}\right)
$$

where $\eta \geq 0,0<\delta<r$ and $\theta<1$. It follows that $S$ solves the stochastic differential equation

$$
d S_{t}=S_{t-}(r-\delta) d t+S_{t-} \eta d W_{t}+S_{t-} \int_{0}^{\infty}\left(e^{\theta y}-1\right)(\mu(d t, d y)-\nu(d t, d y))
$$

and $S_{0}=s$, where $r$ is the riskless interest rate and the dividend rate payed to stockholders is $\delta S_{t}$. Here $\mu(d t, d y)$ is the measure of jumps of the process $J$ with the compensator 
$\nu(d t, d y)=\lambda d t I(y>0) e^{-y} d y$, which means that we work directly under a martingale measure for $S$. Note that the assumption $\theta<1$ guarantees that the jumps of $S$ are integrable under the martingale measure, which is no restriction.

Assume that the firm issues a convertible bond at time zero. At each subsequent time, the bondholder can decide whether to continue to hold the bond, thereby collecting coupons at the rate $c+h S$ with some $c>0$ and $h \geq 0$ fixed, or to convert it into a predetermined number $\gamma>0$ of stocks. On the other hand, at any time the issuing firm can redeem the bond at some call price $K>0$, but at the same time it has to offer the holder to convert the bond instantly. Put differently, the firm can terminate the contract by paying the amount $\max \{K, \gamma S\}$. Thus we can express this contract as a standard game contingent claim. If the holder terminates the contract by converting the bond into $\gamma$ stocks, then the total (discounted) payoff to the holder is given by

$$
L_{t}=\int_{0}^{t} e^{-r u}\left(c+h S_{u}\right) d u+e^{-r t} \gamma S_{t}
$$

while if the issuer terminates the contract, then the total payoff to the holder is given by

$$
U_{t}=\int_{0}^{t} e^{-r u}\left(c+h S_{u}\right) d u+e^{-r t}\left(K \vee \gamma S_{t}\right)
$$

for any time $t \geq 0$. Taking into account that the holder looks for a converting time maximizing the expected discounted payoff, while the issuer looks for a recalling time minimizing the same quantity, we obtain by Kifer [17] or Kallsen and Kühn [16] that an arbitrage-free price for the convertible bond coincides with the value of the corresponding optimal stopping game

$$
\begin{aligned}
V_{*}(s) & =\inf _{\sigma} \sup _{\tau} E_{s}\left[L_{\tau} I(\tau<\sigma)+U_{\sigma} I(\sigma \leq \tau)\right] \\
& =\sup _{\tau} \inf _{\sigma} E_{s}\left[L_{\tau} I(\tau<\sigma)+U_{\sigma} I(\sigma \leq \tau)\right]
\end{aligned}
$$

where the infimum and the supremum are taken over all stopping times $\sigma$ and $\tau$ with respect to the natural filtration of $S$, and $E_{s}$ denotes the expectation under the assumption that $S_{0}=s$ for $s>0$. We also note that when $c \geq r K$ the solution of the problem $(2.5)$ is trivial. Namely, in this case, the upper process (2.4) stopped at the first time when $\gamma S_{t}$ exceeds $K$ is a submartingale, which implies that the issuer should recall the bond 
immediately. So that we assume that $c<r K$. In addition, we suppose that $h<\gamma \delta$, since otherwise the coupon payments for the convertible bond always exceed the dividend payments of the involved stocks.

From the general theory of optimal stopping games (see, e.g., [7], [2]-[3], [8]-[9], [20], $[22],[5])$ it follows that the value function $V_{*}(s)$ of the problem $(2.5)$ lies between $\gamma s$ and $K \vee \gamma s$ for each $s>0$. Then, from the structure of the lower and upper processes (2.3) and (2.4) it is seen that $L_{t}=U_{t}$ when $S_{t} \geq K / \gamma$ for any $t \geq 0$, and hence we have $V_{*}(s)=\gamma s$ for all $s \geq K / \gamma$. Taking into account these facts we will search for optimal stopping times, at which the issuer and holder should terminate the contract, of the form

$$
\begin{aligned}
& \sigma_{*}=\inf \left\{t \geq 0 \mid S_{t} \geq A_{*}\right\} \\
& \tau_{*}=\inf \left\{t \geq 0 \mid S_{t} \geq B_{*}\right\}
\end{aligned}
$$

for some numbers $0<A_{*} \leq K / \gamma$ and $0<B_{*} \leq K / \gamma$ to be determined. We also observe that, by virtue of the structure of the lower and upper gain functions $\gamma s$ and $K \vee \gamma s$, it follows that stopping the game simultaneously by both holder and issuer cannot be optimal as long as the process $S$ fluctuates in the interval $(0, K / \gamma)$. Thus, only the following situations can occur: either $B_{*}<A_{*}=K / \gamma$, or $A_{*}<B_{*}=K / \gamma$, or $A_{*}=B_{*}=K / \gamma$.

By means of standard arguments it can be shown that the infinitesimal operator $\mathbb{L}$ of the process $S$ acts on an arbitrary function $F \in C^{2}(0, \infty)$ (or $F \in C^{1}(0, \infty)$ when $\eta=0$ ) according to the rule

$$
\begin{aligned}
(\mathbb{L} F)(s)= & \left(r-\delta-\frac{\lambda \theta}{1-\theta}\right) s F^{\prime}(s)+\frac{\eta^{2}}{2} s^{2} F^{\prime \prime}(s) \\
& +\int_{0}^{\infty}\left(F\left(s e^{\theta y}\right)-F(s)\right) \lambda e^{-y} d y
\end{aligned}
$$

for all $s>0$. In order to find explicit expressions for the unknown value function $V_{*}(s)$ from (2.5) and the unknown boundaries $A_{*}$ and $B_{*}$ from (2.6)-(2.7) let us use the general theory of optimal stopping problems for continuous time Markov processes (see, e.g., [10] and [29], Chapter III, Section 8, as well as [2]-[3]). We can reduce the optimal stopping 
game (2.5) to the double free-boundary problem

$$
\begin{aligned}
& (\mathbb{L} V-r V)(s)=-(c+h s) \text { for } 0<s<A \wedge B \\
& V(B-)=\gamma B \text { if } B \leq A=\frac{K}{\gamma}, V(A-)=K \vee \gamma A \text { if } A \leq B=\frac{K}{\gamma} \text { (continuous fit) } \\
& V(s)=\gamma s \text { for } s>B \text { if } B \leq A=\frac{K}{\gamma}, V(s)=K \vee \gamma s \text { for } s>A \text { if } A \leq B=\frac{K}{\gamma} \\
& \gamma s<V(s)<K \vee \gamma s \text { for } 0<s<A \wedge B
\end{aligned}
$$

where $0<A \vee B \leq K / \gamma$ and (2.10) play the role of instantaneous-stopping conditions. Moreover, when either $\eta>0$, or $\theta<0$, or $0<\theta<1$ with $r-\delta-\lambda \theta /(1-\theta)>0$ holds, we assume that the conditions

$$
V^{\prime}(B-)=\gamma \text { if } B<A=\frac{K}{\gamma}, V^{\prime}(A-)=0 \text { if } A<B=\frac{K}{\gamma} \text { (smooth fit) }
$$

are satisfied. The latter can be explained by the fact that in the cases mentioned above, leaving the continuation region $\left(0, A_{*} \wedge B_{*}\right)$ the process $S$ can pass through the boundary $A_{*} \wedge B_{*}<K / \gamma$ continuously. This property was earlier observed in [25]-[26] by solving some other optimal stopping problems for jump processes (see also [1] for necessary and sufficient conditions for the occurrence of the smooth-fit condition and references to the related literature).

\section{Solution of the free-boundary problem}

Let us now derive explicit solutions to the free-boundary problem formulated above under different relationships on the parameters of the model.

3.1. Let us first consider the continuous case $\eta>0$ and $\theta=0$. In this case, by means of the same arguments as in [28], Section 8, or [30], Chapter VII, Section 2a, it can be shown that equation (2.9) has the general solution

$$
V(s)=C_{1} s^{\gamma_{1}}+C_{2} s^{\gamma_{2}}+\frac{c}{r}+\frac{h s}{\delta}
$$

where $C_{1}$ and $C_{2}$ are some arbitrary constants, and $\gamma_{2}<0<1<\gamma_{1}$ are given by

$$
\gamma_{i}=\frac{1}{2}-\frac{r-\delta}{\eta^{2}}-(-1)^{i} \sqrt{\left(\frac{1}{2}-\frac{r-\delta}{\eta^{2}}\right)^{2}+\frac{2 r}{\eta^{2}}}
$$


for $i=1,2$. It thus follows that in (3.1) we have $C_{2}=0$, since otherwise $V(s) \rightarrow \pm \infty$ as $s \downarrow 0$, which should be excluded by virtue of the obvious fact that the value function (2.5) is bounded under $s \downarrow 0$. Hence, applying conditions (2.10) and (2.13) to the function (3.1), we get that if $B<A=K / \gamma$ then the following equalities hold

$$
\begin{aligned}
& C_{1} B^{\gamma_{1}}+\frac{c}{r}+\frac{h B}{\delta}=\gamma B \\
& \gamma_{1} C_{1} B^{\gamma_{1}}+\frac{h B}{\delta}=\gamma B
\end{aligned}
$$

and if $A<B=K / \gamma$ then the following equalities hold

$$
\begin{aligned}
& C_{1} A^{\gamma_{1}}+\frac{c}{r}+\frac{h A}{\delta}=K \\
& \gamma_{1} C_{1} A^{\gamma_{1}}+\frac{h A}{\delta}=0 .
\end{aligned}
$$

Thus, solving the systems (3.3)-(3.4) and (3.5)-(3.6) it follows that three regions for $K$ with qualitatively different solutions to the free-boundary problem (besides the trivial solution in case $K \leq c / r)$ can be distinguished. Namely, by means of straightforward calculations we obtain that if the condition

$$
K>\frac{\gamma_{1}}{\gamma_{1}-1} \frac{\gamma \delta}{\gamma \delta-h} \frac{c}{r}
$$

holds then $B_{*}<A_{*}=K / \gamma$ and the solution of system (2.9)-(2.10)+(2.13) is given by

$$
V\left(s ; B_{*}\right)=\frac{1}{\gamma_{1}-1} \frac{c}{r}\left(\frac{s}{B_{*}}\right)^{\gamma_{1}}+\frac{c}{r}+\frac{h s}{\delta}
$$

for all $0<s<B_{*}$ with

$$
B_{*}=\frac{\gamma_{1}}{\gamma_{1}-1} \frac{\delta}{\gamma \delta-h} \frac{c}{r}
$$

if the condition

$$
\frac{\gamma_{1} \gamma \delta}{\gamma_{1}(\gamma \delta-h)+h} \frac{c}{r} \leq K \leq \frac{\gamma_{1}}{\gamma_{1}-1} \frac{\gamma \delta}{\gamma \delta-h} \frac{c}{r}
$$

holds then $A_{*}=B_{*}=K / \gamma$ and the solution of system (2.9)-(2.10) is given by

$$
V(s ; K / \gamma)=\left(\frac{\gamma \delta-h}{\delta} \frac{K}{\gamma}-\frac{c}{r}\right)\left(\frac{\gamma s}{K}\right)^{\gamma_{1}}+\frac{c}{r}+\frac{h s}{\delta}
$$

for all $0<s<K / \gamma$, while if the condition

$$
\frac{c}{r}<K<\frac{\gamma_{1} \gamma \delta}{\gamma_{1}(\gamma \delta-h)+h} \frac{c}{r}
$$


holds then $A_{*}<B_{*}=K / \gamma$ and the solution of system (2.9)-(2.10)+(2.13) is given by

$$
V\left(s ; A_{*}\right)=-\frac{1}{\gamma_{1}-1}\left(K-\frac{c}{r}\right)\left(\frac{s}{A_{*}}\right)^{\gamma_{1}}+\frac{c}{r}+\frac{h s}{\delta}
$$

for all $0<s<A_{*}$ with

$$
A_{*}=\frac{\gamma_{1}}{\gamma_{1}-1} \frac{\delta}{h}\left(K-\frac{c}{r}\right) .
$$

Note that when $h=0$ the condition (3.12) fails to hold, so that $A_{*}<B_{*}=K / \gamma$ cannot occur in that case.

3.2. From now on let us consider the jump-diffusion case $\theta \neq 0$ and for the integrability of jumps assume that $\theta<1$. By means of straightforward calculations we reduce equation (2.9) to the form

$$
-(r+\lambda) V(s)+(r-\delta+\zeta) s V^{\prime}(s)+\frac{\eta^{2}}{2} s^{2} V^{\prime \prime}(s)-\alpha \lambda s^{\alpha} G(s)=-(c+h s)
$$

with $\alpha=1 / \theta$ and $\zeta=-\lambda \theta /(1-\theta)$, where taking into account conditions (2.10)-(2.11) we set

$$
\begin{aligned}
& G(s)=-\int_{s}^{B} V(z) \frac{d z}{z^{\alpha+1}}+\frac{\gamma B^{1-\alpha}}{1-\alpha} \text { if } \alpha=1 / \theta>1 \text { and } B \leq A=\frac{K}{\gamma} \\
& G(s)=-\int_{s}^{A} V(z) \frac{d z}{z^{\alpha+1}}+\frac{\gamma(K / \gamma)^{1-\alpha}}{\alpha(1-\alpha)}-\frac{K A^{-\alpha}}{\alpha} \text { if } \alpha=1 / \theta>1 \text { and } A \leq B=\frac{K}{\gamma} \\
& G(s)=\int_{0}^{s} V(z) \frac{d z}{z^{\alpha+1}} \text { if } \alpha=1 / \theta<0
\end{aligned}
$$

for all $0<s<A \wedge B$. Then, from (3.15) and (3.16)-(3.18) it follows that the function $G(s)$ solves the following (third-order) ordinary differential equation

$$
\begin{aligned}
& \frac{\eta^{2} s^{3}}{2} G^{\prime \prime \prime}(s)+\left[\eta^{2}(\alpha+1)+r-\delta+\zeta\right] s^{2} G^{\prime \prime}(s) \\
& +\left[(\alpha+1)\left(\frac{\eta^{2} \alpha}{2}+r-\delta+\zeta\right)-(r+\lambda)\right] s G^{\prime}(s)-\alpha \lambda G(s)=-s^{-\alpha}(c+h s)
\end{aligned}
$$

for $0<s<A \wedge B$, which has the general solution

$$
G(s)=C_{1} \frac{s^{\beta_{1}}}{\beta_{1}}+C_{2} \frac{s^{\beta_{2}}}{\beta_{2}}+C_{3} \frac{s^{\beta_{3}}}{\beta_{3}}-\frac{c s^{-\alpha}}{r \alpha}+\frac{h s^{1-\alpha}}{\delta(1-\alpha)}
$$

where $C_{1}, C_{2}$ and $C_{3}$ are some arbitrary constants and $\beta_{3}<\beta_{2}<\beta_{1}, \beta_{i} \neq 0$ for $i=1,2,3$, are the real roots of the corresponding (characteristic) equation

$$
\begin{aligned}
& \frac{\eta^{2}}{2} \beta^{3}+\left[\eta^{2}\left(\alpha-\frac{1}{2}\right)+r-\delta+\zeta\right] \beta^{2} \\
& +\left[\alpha\left(\frac{\eta^{2}(\alpha-1)}{2}+r-\delta+\zeta\right)-(r+\lambda)\right] \beta-\alpha \lambda=0 .
\end{aligned}
$$


Therefore, differentiating both sides of the formulas (3.16)-(3.18) we obtain that the integro-differential equation (3.15) has the general solution

$$
V(s)=C_{1} s^{\gamma_{1}}+C_{2} s^{\gamma_{2}}+C_{3} s^{\gamma_{3}}+\frac{c}{r}+\frac{h s}{\delta}
$$

where we set $\gamma_{i}=\beta_{i}+\alpha$ for $i=1,2,3$. Observe that if $\eta=0$ and $r-\delta+\zeta \neq 0$ then it is seen that (3.19) degenerates into a second-order differential equation, and in that case we can put $C_{3}=0$ in (3.20) as well as in (3.22), while the roots of equation (3.21) are explicitly given by

$$
\beta_{i}=\frac{r+\lambda}{2(r-\delta+\zeta)}-\frac{\alpha}{2}-(-1)^{i} \sqrt{\left(\frac{r+\lambda}{2(r-\delta+\zeta)}-\frac{\alpha}{2}\right)^{2}+\frac{\alpha \lambda}{r-\delta+\zeta}}
$$

for $i=1,2$. Note that if $\eta=0$ and $r-\delta+\zeta=0$ then (3.19) degenerates into a first-order differential equation, and in that case we can put $C_{2}=C_{3}=0$ in (3.20) as well as in (3.22), while the unique root of equation (3.21) is given by

$$
\beta_{1}=-\frac{\alpha \lambda}{r+\lambda}
$$

3.2.1. Let us now consider the subcase of negative jumps $\alpha=1 / \theta<0$. Observe from (3.19) that then we have $0<\beta_{2}<-\alpha<1-\alpha<\beta_{1}$ so that $\alpha<\gamma_{2}<0<1<\gamma_{1}$ with $\gamma_{i}=\beta_{i}+\alpha$ for $i=1,2$, and if, in addition, $\eta>0$ then we have $\beta_{3}<0$. It thus follows that in (3.20) as well as in (3.22) we have $C_{2}=C_{3}=0$, since otherwise $G(s) \rightarrow \pm \infty$ and $V(s) \rightarrow \pm \infty$ as $s \downarrow 0$ that should be excluded by virtue of the facts that the value function (2.5) so that the function (3.18) are bounded under $s \downarrow 0$. Therefore, using straightforward calculations we obtain that in the same regions for $K$ as defined in (3.7), (3.10) and (3.12) the solution of system (2.9)-(2.11) $+(2.13)$ is given by the same formulas as in (3.8)-(3.9), (3.11) and (3.13)-(3.14), respectively, with $\gamma_{1}=\beta_{1}+\alpha$, where if $\eta>0$ then $\beta_{1}$ is the largest root of equation (3.21), while if $\eta=0$ then $\beta_{1}$ is given by (3.23).

3.2.2. Let us now consider the subcase of positive jumps $\alpha=1 / \theta>1$. Observe that if, in addition, $\eta>0$ then we have $\beta_{3}<-\alpha<1-\alpha<\beta_{2}<0<\beta_{1}$ so that $\gamma_{3}<0<1<\gamma_{2}<\alpha<\gamma_{1}$ with $\gamma_{i}=\beta_{i}+\alpha$, where $\beta_{i}$ for $i=1,2,3$ are the roots of equation (3.21). It thus follows that in (3.20) as well as in (3.22) we have $C_{3}=0$, since otherwise $V(s) \rightarrow \pm \infty$ as $s \downarrow 0$ that should be excluded by virtue of the fact that the 
function (2.5) is bounded under $s \downarrow 0$. Note that if, in addition, $\eta=0$ and $r-\delta+\zeta>0$ with $\zeta=-\lambda \theta /(1-\theta)$ then we have $1-\alpha<\beta_{2}<0<\beta_{1}$ so that $1<\gamma_{2}<\alpha<\gamma_{1}$ with $\gamma_{i}=\beta_{i}+\alpha$, where $\beta_{i}$ for $i=1,2$ are given by (3.23). Hence, applying conditions (3.16)-(3.17), (2.10) and (2.13) to the functions (3.20) and (3.22), respectively, we get that if $B<A=K / \gamma$ then the following equalities hold

$$
\begin{aligned}
& C_{1} \frac{B^{\gamma_{1}}}{\beta_{1}}+C_{2} \frac{B^{\gamma_{2}}}{\beta_{2}}-\frac{c}{r \alpha}+\frac{h B}{\delta(1-\alpha)}=\frac{\gamma B}{1-\alpha} \\
& C_{1} B^{\gamma_{1}}+C_{2} B^{\gamma_{2}}+\frac{c}{r}+\frac{h B}{\delta}=\gamma B \\
& \gamma_{1} C_{1} B^{\gamma_{1}}+\gamma_{2} C_{2} B^{\gamma_{2}}+\frac{h B}{\delta}=\gamma B
\end{aligned}
$$

and if $A<B=K / \gamma$ then the following equalities hold

$$
\begin{aligned}
& C_{1} \frac{A^{\gamma_{1}}}{\beta_{1}}+C_{2} \frac{A^{\gamma_{2}}}{\beta_{2}}-\frac{c}{r \alpha}+\frac{h A}{\delta(1-\alpha)}=\frac{K}{\alpha(1-\alpha)}\left(\frac{\gamma A}{K}\right)^{\alpha}-\frac{K}{\alpha} \\
& C_{1} A^{\gamma_{1}}+C_{2} A^{\gamma_{2}}+\frac{c}{r}+\frac{h A}{\delta}=K \\
& \gamma_{1} C_{1} A^{\gamma_{1}}+\gamma_{2} C_{2} A^{\gamma_{2}}+\frac{h A}{\delta}=0 .
\end{aligned}
$$

Thus, solving the systems (3.25)-(3.27) and (3.28)-(3.30) we get that in this subcase there are also three regions for $K$ with qualitatively different (nontrivial) solutions to the freeboundary problem. Namely, by means of straightforward calculations we obtain that if the condition

$$
K>\frac{\alpha-1}{\alpha} \frac{\gamma_{1}}{\gamma_{1}-1} \frac{\gamma_{2}}{\gamma_{2}-1} \frac{\gamma \delta}{\gamma \delta-h} \frac{c}{r}
$$

holds then $B_{*}<A_{*}=K / \gamma$ and the solution of system (2.9)-(2.11)+(2.13) is given by

$$
V\left(s ; B_{*}\right)=\frac{1}{\alpha\left(\beta_{1}-\beta_{2}\right)} \frac{c}{r}\left(\frac{\beta_{1} \gamma_{2}}{\gamma_{1}-1}\left(\frac{s}{B_{*}}\right)^{\gamma_{1}}-\frac{\beta_{2} \gamma_{1}}{\gamma_{2}-1}\left(\frac{s}{B_{*}}\right)^{\gamma_{2}}\right)+\frac{c}{r}+\frac{h s}{\delta}
$$

for all $0<s<B_{*}$ with

$$
B_{*}=\frac{\alpha-1}{\alpha} \frac{\gamma_{1}}{\gamma_{1}-1} \frac{\gamma_{2}}{\gamma_{2}-1} \frac{\delta}{\gamma \delta-h} \frac{c}{r}
$$

if the condition

$$
\begin{aligned}
& \frac{\alpha-1}{\alpha} \frac{\gamma_{1} \gamma_{2} \gamma \delta}{\left(\gamma_{1}-1\right)\left(\gamma_{2}-1\right)(\gamma \delta-h)+(\alpha-1) \gamma \delta} \frac{c}{r} \\
& \leq K \leq \frac{\alpha-1}{\alpha} \frac{\gamma_{1}}{\gamma_{1}-1} \frac{\gamma_{2}}{\gamma_{2}-1} \frac{\gamma \delta}{\gamma \delta-h} \frac{c}{r}
\end{aligned}
$$


holds then $A_{*}=B_{*}=K / \gamma$ and the solution of system (2.9)-(2.11) is given by

$$
\begin{aligned}
V(s ; K / \gamma)= & \frac{\beta_{1}}{\beta_{1}-\beta_{2}}\left(\frac{\gamma_{2}-1}{\alpha-1} \frac{\gamma \delta-h}{\delta} \frac{K}{\gamma}-\frac{\gamma_{2}}{\alpha} \frac{c}{r}\right)\left(\frac{\gamma s}{K}\right)^{\gamma_{1}} \\
& -\frac{\beta_{2}}{\beta_{1}-\beta_{2}}\left(\frac{\gamma_{1}-1}{\alpha-1} \frac{\gamma \delta-h}{\delta} \frac{K}{\gamma}-\frac{\gamma_{1}}{\alpha} \frac{c}{r}\right)\left(\frac{\gamma s}{K}\right)^{\gamma_{2}}+\frac{c}{r}+\frac{h s}{\delta}
\end{aligned}
$$

for all $0<s<K / \gamma$, while if the condition

$$
\frac{c}{r}<K<\frac{\alpha-1}{\alpha} \frac{\gamma_{1} \gamma_{2} \gamma \delta}{\left(\gamma_{1}-1\right)\left(\gamma_{2}-1\right)(\gamma \delta-h)+(\alpha-1) \gamma \delta} \frac{c}{r}
$$

holds then $A_{*}<B_{*}=K / \gamma$ and the solution of system (2.9)-(2.11) $+(2.13)$ is given by

$$
\begin{aligned}
V\left(s ; A_{*}\right)= & -\frac{1}{\beta_{1}-\beta_{2}}\left(\gamma_{2}\left(K-\frac{c}{r}\right)-\left(\gamma_{2}-1\right) \frac{h A_{*}}{\delta}\right)\left(\frac{s}{A_{*}}\right)^{\gamma_{1}} \\
& +\frac{1}{\beta_{1}-\beta_{2}}\left(\gamma_{1}\left(K-\frac{c}{r}\right)-\left(\gamma_{1}-1\right) \frac{h A_{*}}{\delta}\right)\left(\frac{s}{A_{*}}\right)^{\gamma_{2}}+\frac{c}{r}+\frac{h s}{\delta}
\end{aligned}
$$

for all $0<s<A_{*}$, where $A_{*}$ is determined as the unique solution of the equation

$$
\left(\frac{\gamma A}{K}\right)^{\alpha}-\frac{\alpha\left(\gamma_{1}-1\right)\left(\gamma_{2}-1\right)}{\beta_{1} \beta_{2}} \frac{h}{\delta} \frac{A}{K}+\frac{(\alpha-1) \gamma_{1} \gamma_{2}}{\beta_{1} \beta_{2} K}\left(K-\frac{c}{r}\right)=0 .
$$

Note that when $h=0$ equation (3.38) admits the explicit solution

$$
A_{*}=\frac{K}{\gamma}\left(\frac{(1-\alpha) \gamma_{1} \gamma_{2}}{\beta_{1} \beta_{2} K}\left(K-\frac{c}{r}\right)\right)^{1 / \alpha}
$$

3.2.3. Let us finally consider the subcase $\eta=0$ and $\alpha=1 / \theta>1$ with $r-\delta+\zeta \leq 0$ and $\zeta=-\lambda \theta /(1-\theta)$. Observe that if, in addition, $r-\delta+\zeta<0$ then we have $\beta_{2}<$ $-\alpha<1-\alpha<\beta_{1}<0$ so that $\gamma_{2}<0<1<\gamma_{1}$ with $\gamma_{i}=\beta_{i}+\alpha$, where $\beta_{i}$ for $i=1,2$ are given by (3.23). It follows that in (3.20) as well as in (3.22) we have $C_{2}=C_{3}=0$, since otherwise $V(s) \rightarrow \pm \infty$ as $s \downarrow 0$ that should be excluded by virtue of the fact that the function (2.5) is bounded under $s \downarrow 0$. Note that if, in addition, $r-\delta+\zeta=0$ then we have $1-\alpha<\beta_{1}<0$ so that $\gamma_{1}>1$ with $\gamma_{1}=\beta_{1}+\alpha$, where $\beta_{1}$ is given by (3.24). Hence, applying conditions (3.16)-(3.17) and (2.10) to the functions (3.20) and (3.22), respectively, we get that if $B \leq A=K / \gamma$ then the following equalities hold

$$
\begin{aligned}
& C_{1} \frac{B^{\gamma_{1}}}{\beta_{1}}-\frac{c}{r \alpha}+\frac{h B}{\delta(1-\alpha)}=\frac{\gamma B}{1-\alpha} \\
& C_{1} B^{\gamma_{1}}+\frac{c}{r}+\frac{h B}{\delta}=\gamma B
\end{aligned}
$$


and if $A \leq B=K / \gamma$ then the following equalities hold

$$
\begin{aligned}
& C_{1} \frac{A^{\gamma_{1}}}{\beta_{1}}-\frac{c}{r \alpha}+\frac{h A}{\delta(1-\alpha)}=\frac{K}{\alpha(1-\alpha)}\left(\frac{\gamma A}{K}\right)^{\alpha}-\frac{K}{\alpha} \\
& C_{1} A^{\gamma_{1}}+\frac{c}{r}+\frac{h A}{\delta}=K .
\end{aligned}
$$

Thus, solving the systems (3.40)-(3.41) and (3.42)-(3.43) we may conclude that, in contrast to the previous parts, in this subcase there are only two regions for $K$ with qualitatively different (nontrivial) solutions to the free-boundary problem. Namely, by means of straightforward calculations we obtain that if the condition

$$
K \geq \frac{\alpha-1}{\alpha} \frac{\gamma_{1}}{\gamma_{1}-1} \frac{\gamma \delta}{\gamma \delta-h} \frac{c}{r}
$$

holds then $B_{*} \leq A_{*}=K / \gamma$ and the solution of system (2.9)-(2.11) is given by

$$
V\left(s ; B_{*}\right)=-\frac{\beta_{1}}{\alpha\left(\gamma_{1}-1\right)} \frac{c}{r}\left(\frac{s}{B_{*}}\right)^{\gamma_{1}}+\frac{c}{r}+\frac{h s}{\delta}
$$

for all $0<s<B_{*}$ with

$$
B_{*}=\frac{\alpha-1}{\alpha} \frac{\gamma_{1}}{\gamma_{1}-1} \frac{\delta}{\gamma \delta-h} \frac{c}{r}
$$

while if the condition

$$
\frac{c}{r}<K \leq \frac{\alpha-1}{\alpha} \frac{\gamma_{1}}{\gamma_{1}-1} \frac{\gamma \delta}{\gamma \delta-h} \frac{c}{r}
$$

holds then $A_{*} \leq B_{*}=K / \gamma$ and the solution of system (2.9)-(2.11) is given by

$$
V\left(s ; A_{*}\right)=\left(K-\frac{c}{r}-\frac{h A_{*}}{\delta}\right)\left(\frac{s}{A_{*}}\right)^{\gamma_{1}}+\frac{c}{r}+\frac{h s}{\delta}
$$

for all $0<s<A_{*}$, where $A_{*}$ is determined as the unique solution of the equation

$$
\left(\frac{\gamma A}{K}\right)^{\alpha}-\frac{\alpha\left(\gamma_{1}-1\right)}{\beta_{1}} \frac{h}{\delta} \frac{A}{K}+\frac{(\alpha-1) \gamma_{1}}{\beta_{1} K}\left(K-\frac{c}{r}\right)=0 .
$$

Note that when $h=0$ equation (3.49) admits the explicit solution

$$
A_{*}=\frac{K}{\gamma}\left(\frac{(1-\alpha) \gamma_{1}}{\beta_{1} K}\left(K-\frac{c}{r}\right)\right)^{1 / \alpha}
$$

Remark that in this case the smooth-fit conditions (2.13) fail to hold, that can be explained by the fact that when $\eta=0$ and $0<\theta<1$ with $r-\delta-\lambda \theta /(1-\theta) \leq 0$, leaving the continuation region $\left(0, A_{*} \wedge B_{*}\right)$ the process $S$ can pass through the boundary $A_{*} \wedge B_{*}<$ 
$K / \gamma$ only by jumping. Such an effect was earlier observed in [25]-[26] by solving some other optimal stopping problems for jump processes. According to the results in [1] the smooth-fit condition can fail to hold because the compound Poisson process $J$ has finite variation.

\section{Main result and proof}

Taking into account the facts proved above, let us now formulate the main assertion of the paper.

Theorem 4.1. Let the process $S$ be given by (2.1) and assume that the standing assumptions $0<\delta<r$ and $0 \leq h<\gamma \delta$ hold. Then the value function of the problem (2.5) admits the representation

$$
V_{*}(s)= \begin{cases}V\left(s ; A_{*} \wedge B_{*}\right), & \text { if } 0<s<A_{*} \wedge B_{*} \\ \gamma s, & \text { if } B_{*} \leq A_{*} \text { and } s \geq B_{*} \\ K \vee \gamma s, & \text { if } A_{*} \leq B_{*} \text { and } s \geq A_{*}\end{cases}
$$

and the optimal stopping times $\sigma_{*}$ and $\tau_{*}$ have the structure (2.6)-(2.7), where the function $V\left(s ; A_{*} \wedge B_{*}\right)$ and the boundaries $A_{*}$ and $B_{*}$ are specified as follows:

(i) if $\eta>0$ and $\theta=0$ then under condition (3.7) we have $B_{*}<A_{*}=K / \gamma$ and $V\left(s ; B_{*}\right)$ is given by (3.8) with $B_{*}$ from (3.9), under condition (3.10) we have $A_{*}=B_{*}=K / \gamma$ and $V(s ; K / \gamma)$ is given by (3.11), while under condition (3.12) we have $A_{*}<B_{*}=K / \gamma$ and $V\left(s ; A_{*}\right)$ is given by (3.13) with $A_{*}$ being the unique solution of (3.14), where $\gamma_{1}$ is given by (3.2);

(ii) if $\theta<0$ then the assertion (i) holds with $\gamma_{1}$ replaced by $\beta_{1}+1 / \theta$, where $\beta_{1}$ is the largest root of equation (3.21) in case $\eta>0$, while $\beta_{1}$ is given by (3.23) in case $\eta=0$;

(iii) if $\eta>0$ and $0<\theta<1$ then under condition (3.31) we have $B_{*}<A_{*}=K / \gamma$ and $V\left(s ; B_{*}\right)$ is given by (3.32) with $B_{*}$ from (3.33), under condition (3.34) we have $A_{*}=B_{*}=K / \gamma$ and $V(s ; K / \gamma)$ is given by (3.35), while under condition (3.36) we have $A_{*}<B_{*}=K / \gamma$ and $V\left(s ; A_{*}\right)$ is given by (3.37) with $A_{*}$ being the unique solution of (3.38), where $\gamma_{i}=\beta_{i}+1 / \theta$ and $\beta_{i}$ for $i=1,2$ are the two largest roots of equation (3.21); 
(iv) if $\eta=0$ and $0<\theta<1$ with $r-\delta-\lambda \theta /(1-\theta)>0$ then the assertion (iii) holds with $\beta_{i}$ for $i=1,2$ given by (3.23);

(v) if $\eta=0$ and $0<\theta<1$ with $r-\delta-\lambda \theta /(1-\theta) \leq 0$ then under condition (3.44) we have $B_{*} \leq A_{*}=K / \gamma$ and $V\left(s ; B_{*}\right)$ is given by (3.45) with $B_{*}$ from (3.46), while under condition (3.47) we have $A_{*} \leq B_{*}=K / \gamma$ and $V\left(s ; A_{*}\right)$ is given by (3.48) with $A_{*}$ being the unique solution of (3.49), where $\gamma_{1}=\beta_{1}+1 / \theta$ and $\beta_{1}$ is given by (3.23) in case $r-\delta-\lambda \theta /(1-\theta)<0$, while $\beta_{1}$ is given by (3.24) in case $r-\delta-\lambda \theta /(1-\theta)=0$.

Proof. In order to verify the assertions stated above, it remains to show that the function (4.1) coincides with the value function (2.5) and the stopping times $\sigma_{*}$ and $\tau_{*}$ from (2.6)-(2.7) with the boundaries $A_{*}$ and $B_{*}$ specified above are optimal. For this, let us denote by $V(s)$ the right-hand side of the expression (4.1). In this case, by means of straightforward calculations and the assumptions above it follows that the function $V(s)$ solves the system (2.9)-(2.11), and conditions (2.13) are satisfied when either $\eta>0$, or $\theta<0$, or $0<\theta<1$ with $r-\delta-\lambda \theta /(1-\theta)>0$ holds. In addition, we note that $V(s)$ can be written as a difference of two convex functions. Then, applying Itô-Tanaka-Meyer formula (see, e.g., [13], Chapter V, Theorem 5.52, or [27], Chapter IV, Theorem 51) to $e^{-r t} V\left(S_{t}\right)$, we obtain

$$
\begin{aligned}
& e^{-r t} V\left(S_{t}\right) \\
& =V(s)+\int_{0}^{t} e^{-r u}(\mathbb{L} V-r V)\left(S_{u}\right) I\left(S_{u} \neq A_{*}, S_{u} \neq B_{*}, S_{u} \neq K / \gamma\right) d u \\
& \quad+M_{t}+\frac{1}{2} \int_{0}^{t} e^{-r u}\left(V^{\prime}(K / \gamma+)-V^{\prime}(K / \gamma-)\right) I\left(S_{u}=K / \gamma\right) d \ell_{u}
\end{aligned}
$$

where the process $\left(\ell_{t}\right)_{t \geq 0}$, the local time of $S$ at the point $K / \gamma$, is given by

$$
\ell_{t}=P_{s}-\lim _{\varepsilon \downarrow 0} \frac{1}{2 \varepsilon} \int_{0}^{t} I\left(K / \gamma-\varepsilon<S_{u}<K / \gamma+\varepsilon\right) \eta^{2} S_{u}^{2} d u
$$

(see, e.g., [27], page 178, Corollary 3), and the process $\left(M_{t}\right)_{t \geq 0}$ given by

$$
\begin{aligned}
M_{t}= & \int_{0}^{t} e^{-r u} V^{\prime}\left(S_{u}\right) I\left(S_{u} \neq A_{*}, S_{u} \neq B_{*}, S_{u} \neq K / \gamma\right) \eta S_{u} d W_{u} \\
& +\int_{0}^{t} \int_{0}^{\infty} e^{-r u}\left(V\left(S_{u-} e^{\theta y}\right)-V\left(S_{u-}\right)\right)(\mu(d u, d y)-\nu(d u, d y))
\end{aligned}
$$


is a local martingale with respect to $P_{s}$ being a probability measure under which the process $S$ starts at $0<s<K / \gamma$. Note that when $\eta=0$ and $r-\delta-\lambda \theta /(1-\theta)=0$, the indicators in the formulas (4.2) and (4.4) can be set to one.

By the construction of $V(s)$ in the previous section and using straightforward calculations it can be verified that $(\mathbb{L} V-r V)(s) \leq-(c+h s)$ for all $0<s<A_{*}$ and $(\mathbb{L} V-r V)(s) \geq-(c+h s)$ for all $0<s<B_{*}$. Moreover, by means of standard arguments it can be shown that for the derivative we have $0 \leq V^{\prime}\left(s ; A_{*} \wedge B_{*}\right) \leq \gamma$ on the interval $\left(0, A_{*} \wedge B_{*}\right)$, and thus the property (2.12) also holds that together with (2.10)-(2.11) yields $V(s) \geq \gamma s$ and $V(s) \leq K \vee \gamma s$ for all $s>0$. Observe that from (2.1) it is seen that when either $\eta>0$ or $r-\delta-\lambda \theta /(1-\theta) \neq 0$, the time spent by the process $S$ at the points $A_{*}, B_{*}$ and $K / \gamma$ is of Lebesgue measure zero. Thus, in those cases, the indicator appearing in the first integral of the expression (4.2) can be ignored. Hence, from (4.2) and the structure of the stopping times in (2.6)-(2.7) with $0<A_{*} \leq K / \gamma$ and $0<B_{*} \leq K / \gamma$ it follows that

$$
\begin{aligned}
& L_{\sigma_{*} \wedge \tau} \leq \int_{0}^{\sigma_{*} \wedge \tau} e^{-r u}\left(c+h S_{u}\right) d u+e^{-r\left(\sigma_{*} \wedge \tau\right)} V\left(S_{\sigma_{*} \wedge \tau}\right) \leq V(s)+M_{\sigma_{*} \wedge \tau} \\
& U_{\sigma \wedge \tau_{*}} \geq \int_{0}^{\sigma \wedge \tau_{*}} e^{-r u}\left(c+h S_{u}\right) d u+e^{-r\left(\sigma \wedge \tau_{*}\right)} V\left(S_{\sigma \wedge \tau_{*}}\right) \geq V(s)+M_{\sigma \wedge \tau_{*}}
\end{aligned}
$$

for any stopping times $\sigma$ and $\tau$ of the process $S$ started at $0<s<K / \gamma$.

Let $\left(\tau_{n}\right)_{n \in \mathbb{N}}$ be an arbitrary localizing sequence of stopping times for the process $\left(M_{t}\right)_{t \geq 0}$. Then using (4.5)-(4.6) and the fact that, by construction, we have $V\left(S_{\sigma_{*}}\right)=$ $K \vee \gamma S_{\sigma_{*}}$ and $V\left(S_{\tau_{*}}\right)=\gamma S_{\tau_{*}}$, respectively, by means of the optional sampling theorem (see, e.g., [14], Chapter I, Theorem 1.39) we get

$$
\begin{aligned}
& E_{s}\left[L_{\tau \wedge \tau_{n}} I\left(\tau \wedge \tau_{n}<\sigma_{*}\right)+U_{\sigma_{*}} I\left(\sigma_{*} \leq \tau \wedge \tau_{n}\right)\right] \\
& \leq E_{s}\left[\int_{0}^{\sigma_{*} \wedge \tau \wedge \tau_{n}} e^{-r u}\left(c+h S_{u}\right) d u+e^{-r\left(\sigma_{*} \wedge \tau \wedge \tau_{n}\right)} V\left(S_{\sigma_{*} \wedge \tau \wedge \tau_{n}}\right)\right] \\
& \leq V(s)+E_{s}\left[M_{\sigma_{*} \wedge \tau \wedge \tau_{n}}\right]=V(s) \\
& E_{s}\left[L_{\tau_{*}} I\left(\tau_{*}<\sigma \wedge \tau_{n}\right)+U_{\sigma \wedge \tau_{n}} I\left(\sigma \wedge \tau_{n} \leq \tau_{*}\right)\right] \\
& \geq E_{s}\left[\int_{0}^{\sigma \wedge \tau_{*} \wedge \tau_{n}} e^{-r u}\left(c+h S_{u}\right) d u+e^{-r\left(\sigma \wedge \tau_{*} \wedge \tau_{n}\right)} V\left(S_{\sigma \wedge \tau_{*} \wedge \tau_{n}}\right)\right] \\
& \geq V(s)+E_{s}\left[M_{\sigma \wedge \tau_{*} \wedge \tau_{n}}\right]=V(s)
\end{aligned}
$$


for all $0<s<K / \gamma$. Taking into account the structure of the processes in (2.3)-(2.4) and the stopping times in (2.6)-(2.7) as well as the integrability of jumps of the process $S$, it can be shown that the property

$$
E_{s}\left[\sup _{t \geq 0} L_{\left(\sigma_{*} \vee \tau_{*}\right) \wedge t}\right] \leq E_{s}\left[\sup _{t \geq 0} U_{\left(\sigma_{*} \vee \tau_{*}\right) \wedge t}\right]<\infty
$$

holds for all $0<s<K / \gamma$ and the variables $L_{\sigma_{*} \vee \tau_{*}}$ and $U_{\sigma_{*} \vee \tau_{*}}$ are bounded on the set $\left\{\sigma_{*} \vee \tau_{*}=\infty\right\}$. Hence, letting $n$ go to infinity in (4.7)-(4.8) and using Fatou's lemma, we obtain that for any stopping times $\sigma$ and $\tau$ the inequalities

$$
\begin{aligned}
& E_{s}\left[L_{\tau} I\left(\tau<\sigma_{*}\right)+U_{\sigma_{*}} I\left(\sigma_{*} \leq \tau\right)\right] \\
& \leq V(s) \leq E_{s}\left[L_{\tau_{*}} I\left(\tau_{*}<\sigma\right)+U_{\sigma} I\left(\sigma \leq \tau_{*}\right)\right]
\end{aligned}
$$

are satisfied for all $0<s<K / \gamma$, from where the desired assertion follows directly. Inserting $\sigma_{*}$ in place of $\sigma$ and $\tau_{*}$ in place of $\tau$ into (4.10), we obtain that the equality

$$
E_{s}\left[L_{\tau_{*}} I\left(\tau_{*}<\sigma_{*}\right)+U_{\sigma_{*}} I\left(\sigma_{*} \leq \tau_{*}\right)\right]=V(s)
$$

holds for all $0<s<K / \gamma$.

Remark 4.2. Let us assume that $h=0$. In this case, if $\theta \leq 0$ then condition (3.12) never holds, so that the situation $A_{*}<B_{*}=K / \gamma$ cannot occur. On the other hand, if $0<\theta<1$ then conditions (3.36) and (3.47) may be satisfied, and thus the situation $A_{*}<B_{*}=K / \gamma$ turns out to be possible. This shows that the occurrence of positive jumps in the stock price process $S$ may lead to a change in the structure of optimal stopping strategies for the problem (2.5).

Remark 4.3. Let us assume that $\eta=0$ and $0<\theta<1$ with $r-\delta-\lambda \theta /(1-\theta) \leq 0$. In this case there is a single recall price $K$ satisfying both (3.44) and (3.47) such that the situation $A_{*}=B_{*}=K / \gamma$ occurs (signifying that the stock price process $S$ should not be stopped before it exceeds the level $K / \gamma)$. This stays in contrast to the case when either $\eta>0$ or $r-\delta-\lambda \theta /(1-\theta)>0$ holds, where, in general, a whole interval of recall prices $K$ can exist for which $A_{*}=B_{*}=K / \gamma$ is the solution (see (3.10) or (3.34)). 


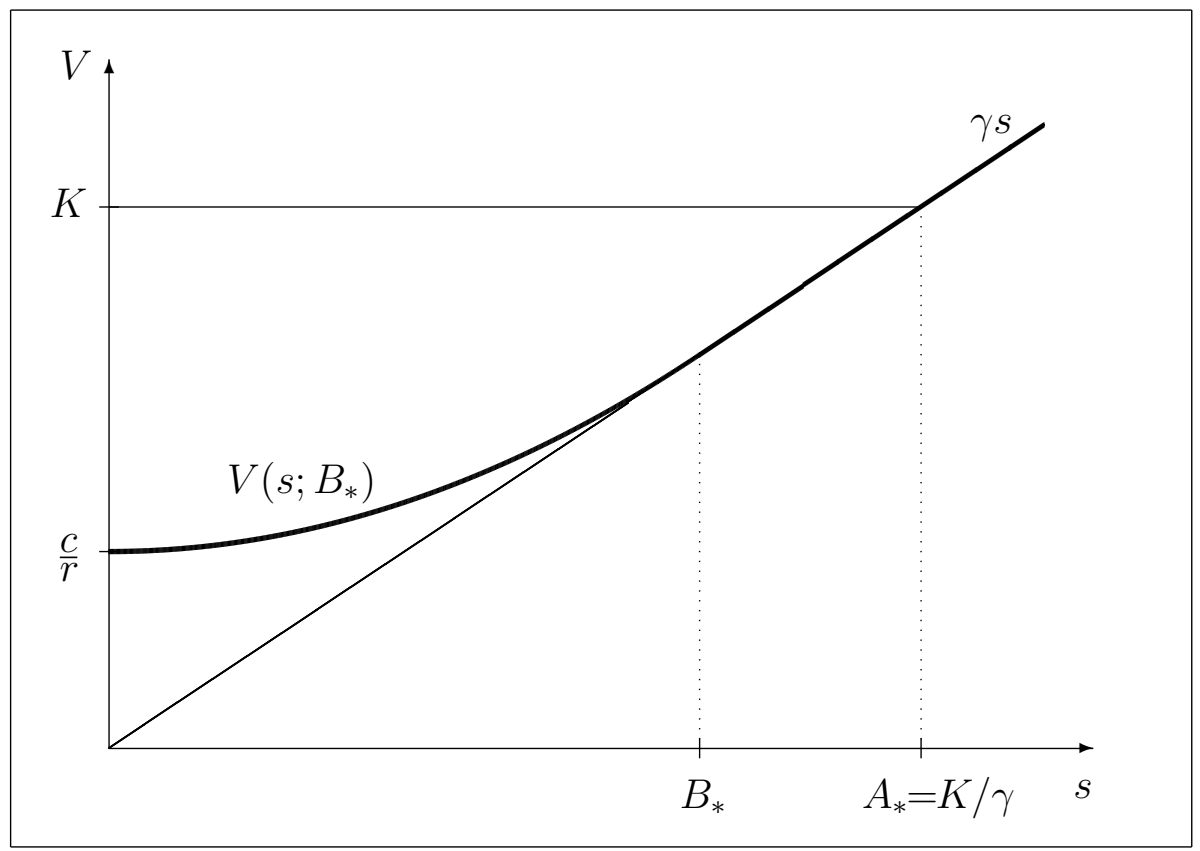

Figure 1. A computer drawing of the value function $V_{*}(s)$ in the situation $B_{*}<A_{*}=K / \gamma$

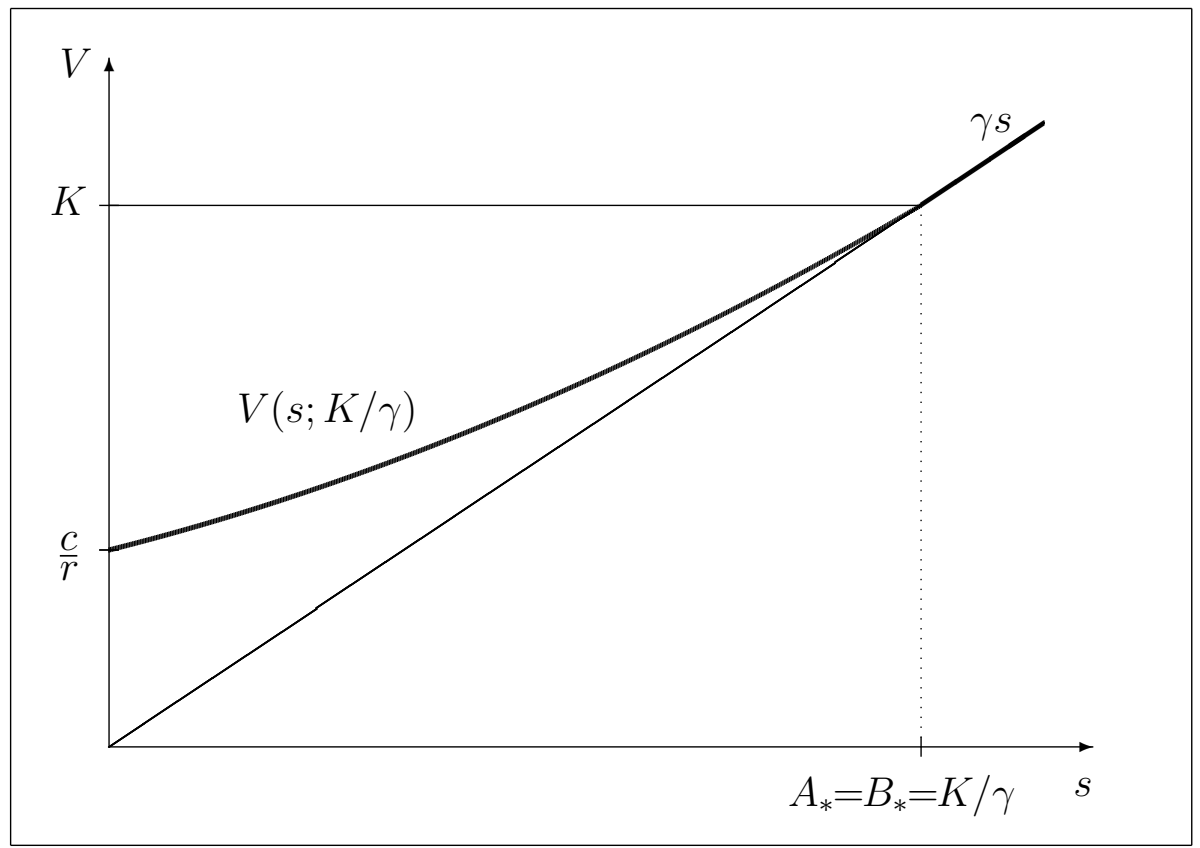

Figure 2. A computer drawing of the value function $V_{*}(s)$ in the situation $A_{*}=B_{*}=K / \gamma$ 


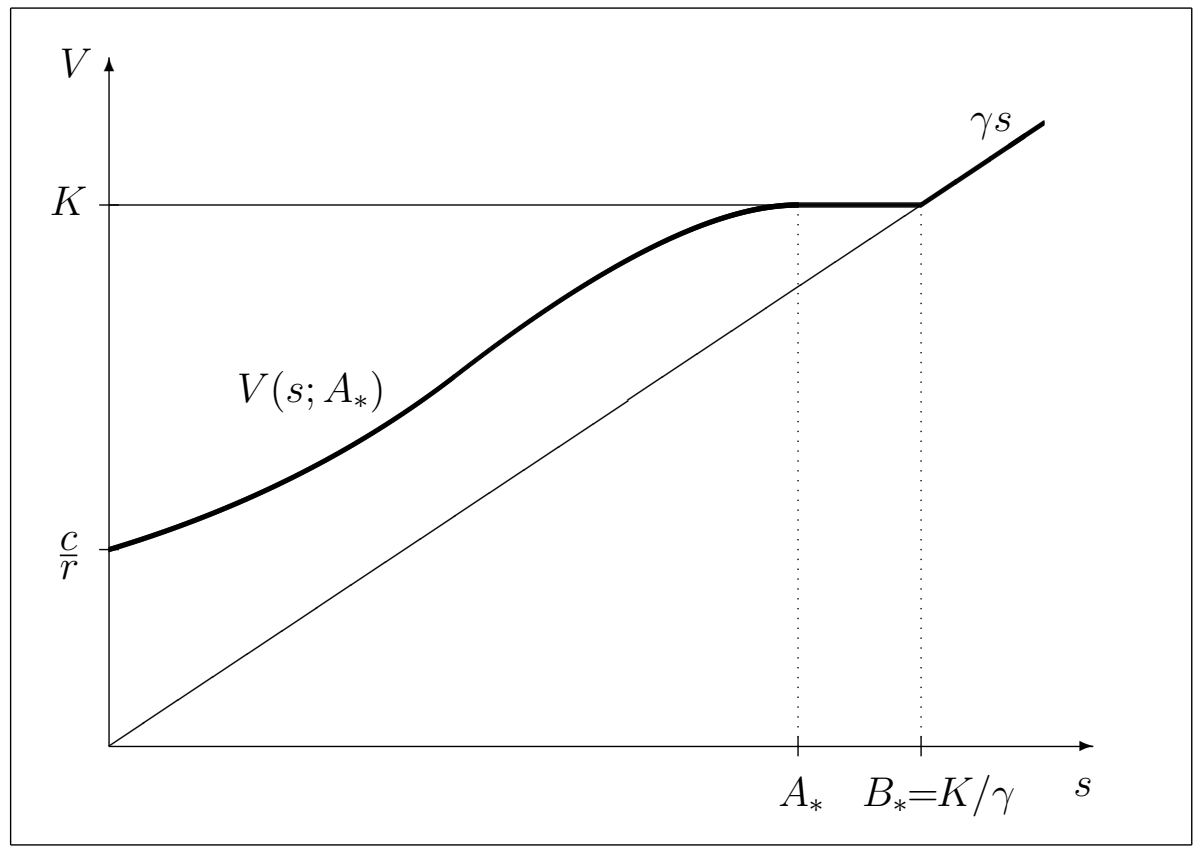

Figure 3. A computer drawing of the value function $V_{*}(s)$ in the situation $A_{*}<B_{*}=K / \gamma$ with smooth fit

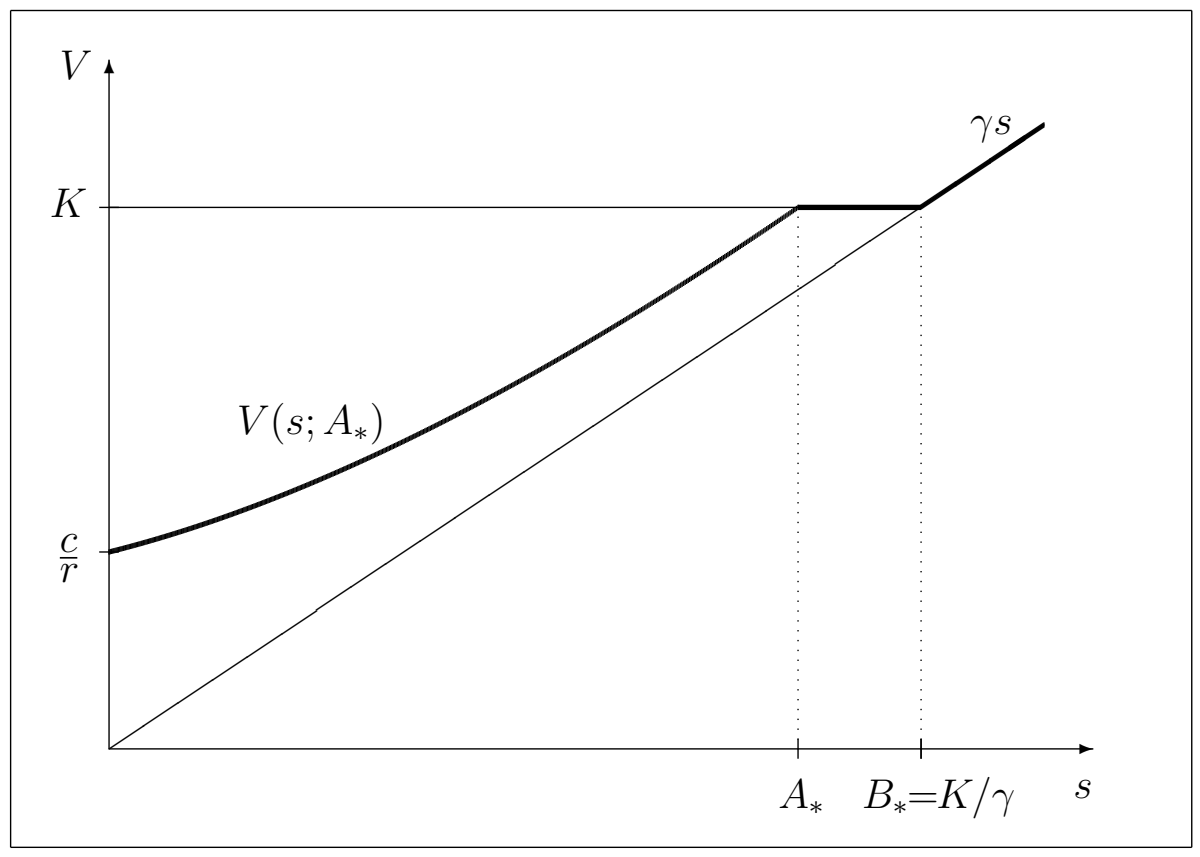

Figure 4. A computer drawing of the value function $V_{*}(s)$ in the situation $A_{*}<B_{*}=K / \gamma$ without smooth fit 


\section{Conclusion}

We have considered the convertible (callable) bond optimal stopping game in a jumpdiffusion model with infinite time horizon. For continuous models, in the context of a firm value approach, the articles of Brennan and Schwartz [4] and Ingersoll [11]-[12] as well as the recent results of Sîrbu, Pikovsky and Shreve [31] tell us that under the standing assumption that the coupon payments are less than the interest rate on the recall price, that is $c<r K$ (in the case $h=0$ ), the issuer never recalls the bond before the conversion value exceeds the recall price. This means that the initial optimal stopping game can be reduced to an optimal conversion problem. We recover this phenomenon for a reduced form model when the stock price can possess negative jumps (see Fig. 1). We note that in that case there is also a whole interval of recall prices $K$ such that the stock price process reaches the level $K / \gamma$ before being stopped by the holder or issuer (see Fig. 2).

In addition, in this paper it is also shown that the occurrence of positive jumps in the stock price process can cause the issuer to recall the bond earlier, that is even if $\gamma s<K$. The reason for this is the risk that the conversion value can jump over $K$ that may lead to a higher payoff $\gamma s>K$. If there is either a diffusion term or a positive drift term, then the smooth-fit condition at the optimal boundary holds (see Fig. 3). An extreme situation can occur when the diffusion term vanishes and the price process merely consists of positive jumps and a negative drift. In that case, the issuer may recall the bond earlier and the smooth-fit condition fails to hold (see Fig. 4).

Acknowledgments. Parts of this research were carried out while the first author was visiting the Goethe-University Frankfurt. Financial support by the Frankfurt MathFinance Institute is gratefully acknowledged. The authors want to thank both anonymous referees for valuable comments.

\section{References}

[1] Alili, L. and Kyprianou, A. E. (2004). Some remarks on first passage of Lévy processes, the American put and pasting principles. To appear in Ann. Appl. 
Probab.

[2] Bensoussan, A and Friedman, A. (1974). Non-linear variational inequalities and differential games with stopping times. J. Funct. Anal. 16, 305-352.

[3] Bensoussan, A and Friedman, A. (1977). Nonzero-sum stochastic differential games with stopping times and free-boundary problems. Trans. Amer. Math. Soc. 231, 275-327.

[4] Brennan, M. J. and Schwartz, E. S. (1977). Convertible bonds: valuation and optimal strategies for call and conversion. Journal of Finance 32, 1699-1715.

[5] Cvitanić, J. and Karatzas, I. (1996). Backward stochastic differential equations with reflection and Dynkin games. Ann. Probab. 24, 2024-2056.

[6] Davis, M. H. A. and Lischka, F. R. (1999). Convertible bonds with market risk and credit risk. Preprint.

[7] Dynkin, E. B. (1969). Game variant of a problem on optimal stopping. Soviet Math. Dokl. 10, 270-274.

[8] Friedman, A. (1973). Stochastic games and variational inequalities. Arch. Rational Mech. Anal. 51, 321-346.

[9] Friedman, A. (1976). Stochastic Differential Equations and Applications II. Academic Press, New York.

[10] Grigelionis, B. I. and Shiryaev, A. N. (1966). On Stefan's problem and optimal stopping rules for Markov processes. Theory Probab. Appl. 11, 541-558.

[11] Ingersoll, J. E. (1977). A contingent-claims valuation of convertible securities. Journal of Financial Economics 4, 289-322.

[12] Ingersoll, J. E. (1977). An examination of corporate call policies on convertible securities. Journal of Finance 32, 463-478. 
[13] Jacod, J. (1979). Calcul Stochastique et Problèmes de Martingales. Lecture Notes in Mathematics, Berlin.

[14] Jacod, J. and ShiRyaev, A. N. (1987). Limit Theorems for Stochastic Processes. Springer, Berlin.

[15] Kallsen, J. and Kühn, C. (2004). Pricing derivatives of American and game type in incomplete markets. Finance $\mathscr{E}$ Stochastics 8, 261-284.

[16] Kallsen, J. and KüHn, C. (2005). Convertible bonds: financial derivatives of game type. EURANDOM book on Exotic Option Pricing under Advanced Lévy Models, editors: A. Kyprianou, W. Schoutens, and P. Wilmott. Wiley, Chichester.

[17] Kifer, Y. (2000). Game options. Finance \& Stochastics 4, 443-463.

[18] Kou, S. G. (2002). A jump diffusion model for option pricing. Management Science 48, 1086-1101.

[19] Kou, S. G. and Wang, H. (2004). Option pricing under a double exponential jump diffusion model. Management Science 50, 1178-1192.

[20] Krylov, N. V. (1971). Control of Markov processes and W-spaces. Izvestija 5, 233-266.

[21] Kyprianou, A. E. (2004). Some calculations for Israeli options. Finance 86 Stochastics 8, 73-86.

[22] Lepeltier, J. P. and Mainguenau, M. A. (1984). Le jeu de Dynkin en théorie générale sans l'hypothèse de Mokobodski. Stochastics 13, 25-44.

[23] Mordecki, E. (1999). Optimal stopping for a diffusion with jumps. Finance 86 Stochastics 3, 227-236.

[24] Mordecki, E. (2002). Optimal stopping and perpetual options for Lévy processes. Finance 85 Stochastics 6, 473-493. 
[25] Peskir, G. and Shiryaev, A. N. (2000). Sequential testing problems for Poisson processes. Ann. Statist. 28, 837-859.

[26] Peskir, G. and Shiryaev, A. N. (2002). Solving the Poisson disorder problem. Advances in Finance and Stochastics. Essays in Honour of Dieter Sondermann. Sandmann, K. and Schönbucher, P. eds. Springer, 295-312.

[27] Protter, PH. (1990). Stochastic Integration and Differential Equations. Springer, New York.

[28] Shiryaev, A. N., Kabanov, Y. M., Kramkov, D. O. and MelNIKOV, A. V. (1994). On the pricing of options of European and American types, II. Continuous time. Theory Probab. Appl. 39, 61-102.

[29] ShIRyaev, A. N. (1978). Optimal Stopping Rules. Springer, Berlin.

[30] Shiryaev, A. N. (1999). Essentials of Stochastic Finance. World Scientific, Singapore.

[31] Sîrbu, M., Pikovsky, I. and Shreve, S. (2004). Perpetual Convertible Bonds. SIAM J. Control. Optim. 43, 58-85.

Pavel V. Gapeev

Russian Academy of Sciences

Institute of Control Sciences

Profsoyuznaya Str. 65

117997 Moscow, Russia

e-mail: gapeev@cniica.ru

Christoph Kühn

Johann Wolfgang Goethe-University

Frankfurt MathFinance Institute

D-60054 Frankfurt a.M., Germany

e-mail: ckuehn@math.uni-frankfurt.de 